\title{
QAGUPUBLICATIONS
}

Space Weather: The International Journal of Research and Applications

Supporting Information for

\section{Validation of Ionospheric Specifications During Geomagnetic Storms: TEC and foF2 during the 2013 March Storm Event}

J. S. Shim ${ }^{1 *}$, I. Tsagouri ${ }^{2}$, L. Goncharenko ${ }^{3}$, L. Rastaetter ${ }^{4}$, M. Kuznetsova ${ }^{4}$, D. Bilitza ${ }^{5}$, M. Codrescu ${ }^{6}$, A. J. Coster ${ }^{3}$, S. C. Solomon ${ }^{7}$, M. Fedrizzi ${ }^{6}$, M. Förster ${ }^{8}$, T. J. FullerRowell $^{6}$, L. C. Gardner ${ }^{9}$, J. Huba ${ }^{10}$, A. A. Namgaladze ${ }^{11}$, B. E. Prokhorov ${ }^{8}$, A. J. Ridley ${ }^{12}$, L. Scherliess ${ }^{9}$, R. W. Schunk ${ }^{9}$, J. J. Sojka ${ }^{9}$, L. Zhu ${ }^{9}$

1. The Catholic University of America, NASA GSFC, Greenbelt, MD, USA,

2. National Observatory of Athens, Penteli, Greece

3. Haystack Observatory, Westford, MA, USA,

4. NASA GSFC, Greenbelt, MD, USA,

5. Department of Physics and Astronomy, George Mason University, Fairfax, Virginia, USA,

6. NOAA SWPC, Boulder, CO, USA,

7. High Altitude Observatory, NCAR, Boulder, CO, USA,

8. Helmholtz Centre Potsdam, GFZ German Research Centre for Geosciences, Potsdam, Germany,

9. Utah State Univ. Logan, UT, USA

10. Plasma Physics Division, Naval Research Laboratory, Washington, D. C., USA,

11. Murmansk Arctic State University, Murmansk, Russia,

12. Space Physics Research Laboratory, Univ. of Michigan, Ann Arbor, MI, USA,

\section{Contents of this file}

Figures S1 S3

\section{Introduction}

This supporting information file includes:

1. Figure S1: Comparison foF 2 and TEC data with modeled values

2. Figure S2: Scatter plots of observed and modeled foF2

3. Figure S3: RMSE scatter plots

4. Figure S4: Yield and Timing Error scatter plots 
4 IR

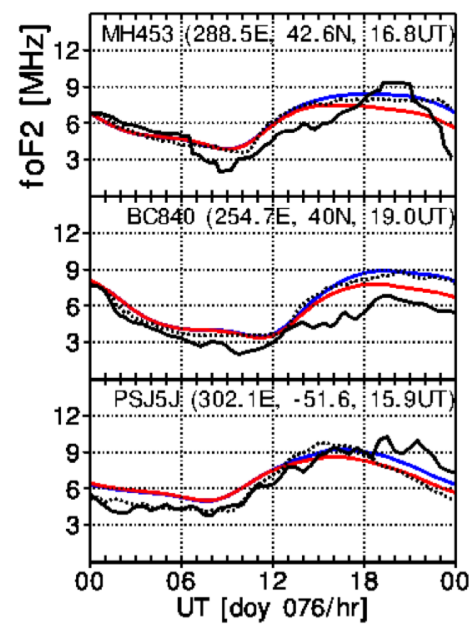

1 IFM

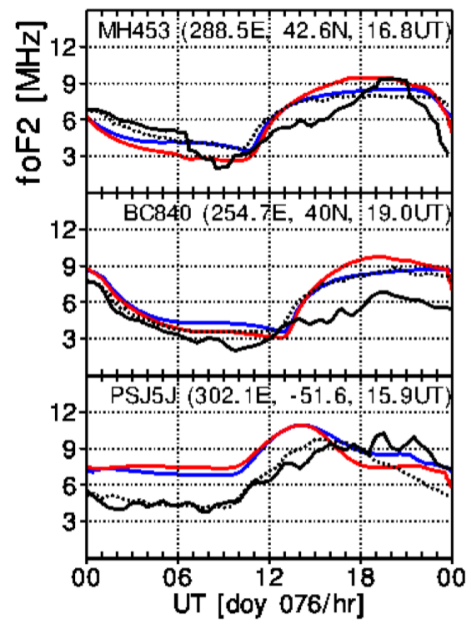

1_SAMI3

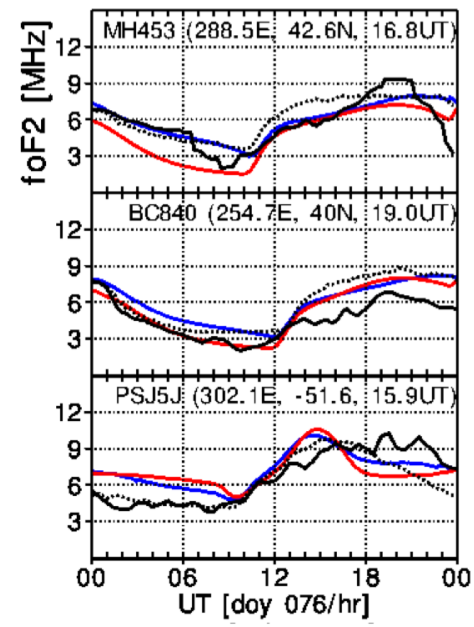

(a)

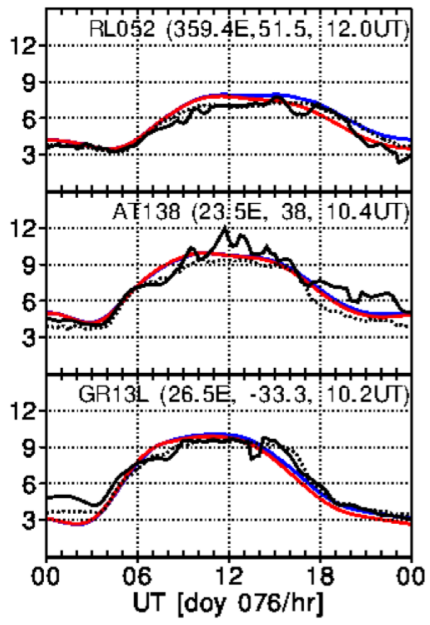

(b)

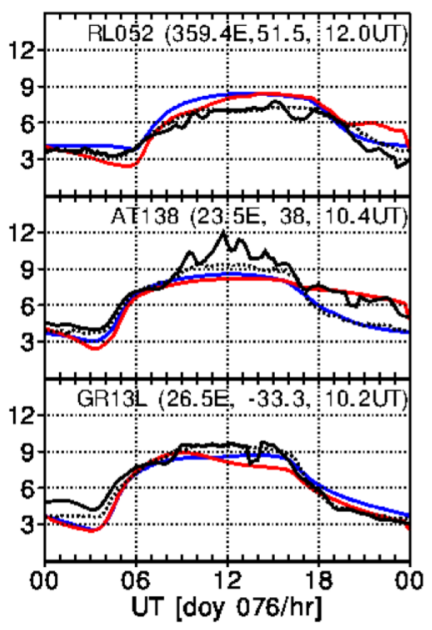

(c)

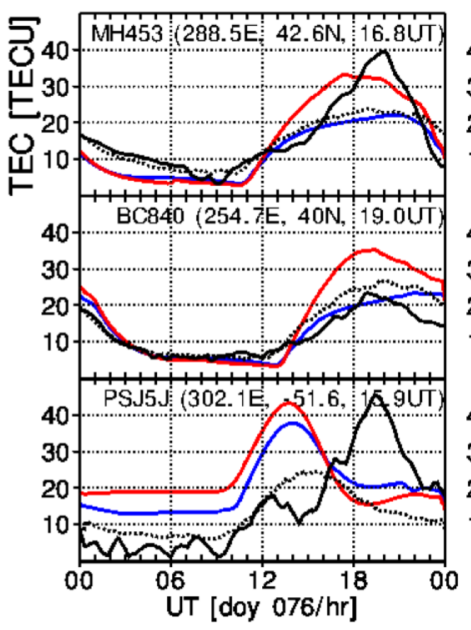

1_IFM

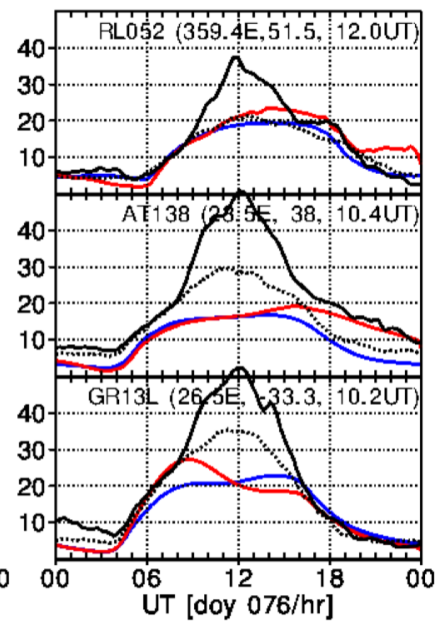

1 SAMI3

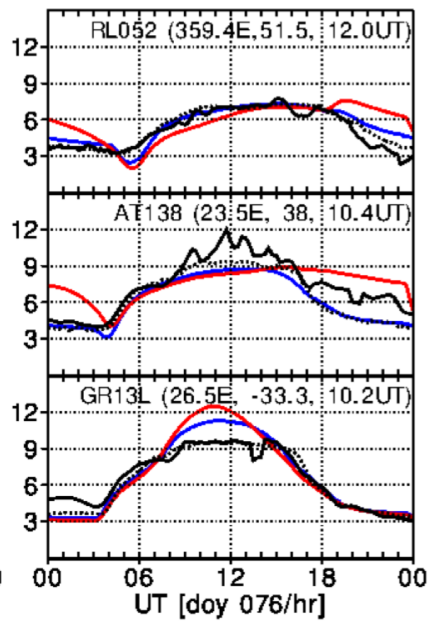

4 IRI
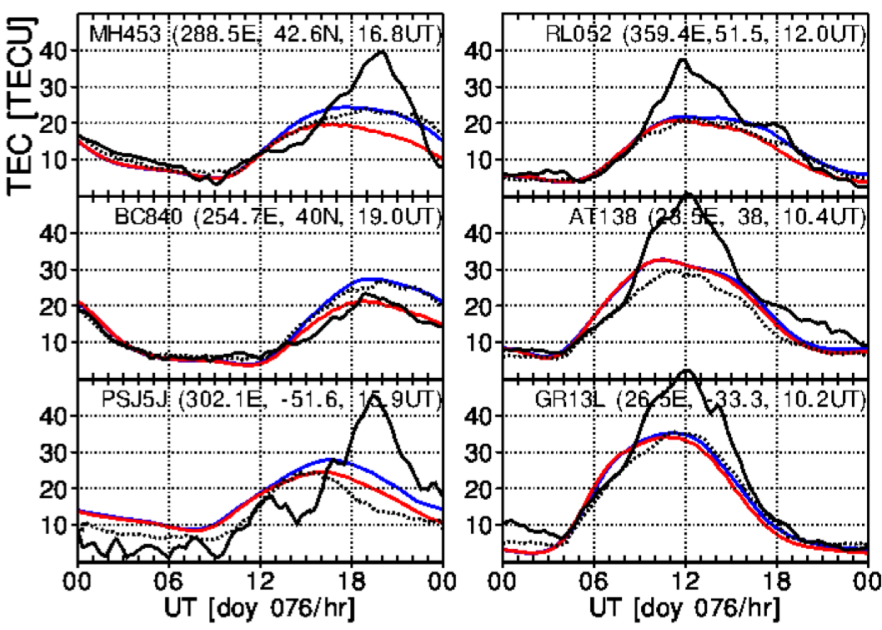

UT [doy 076/hr] 


\section{1_CTIPE}
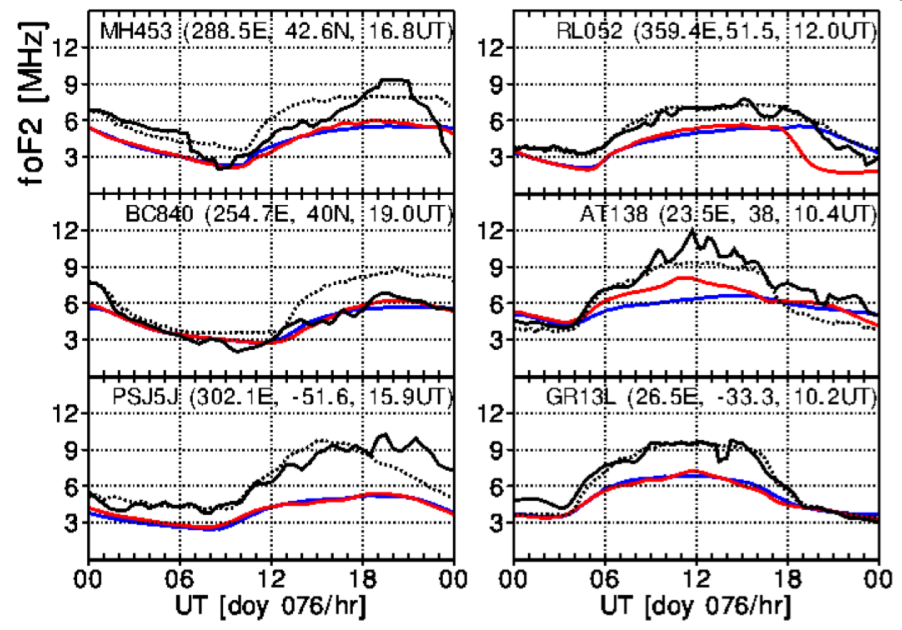

6_GITM
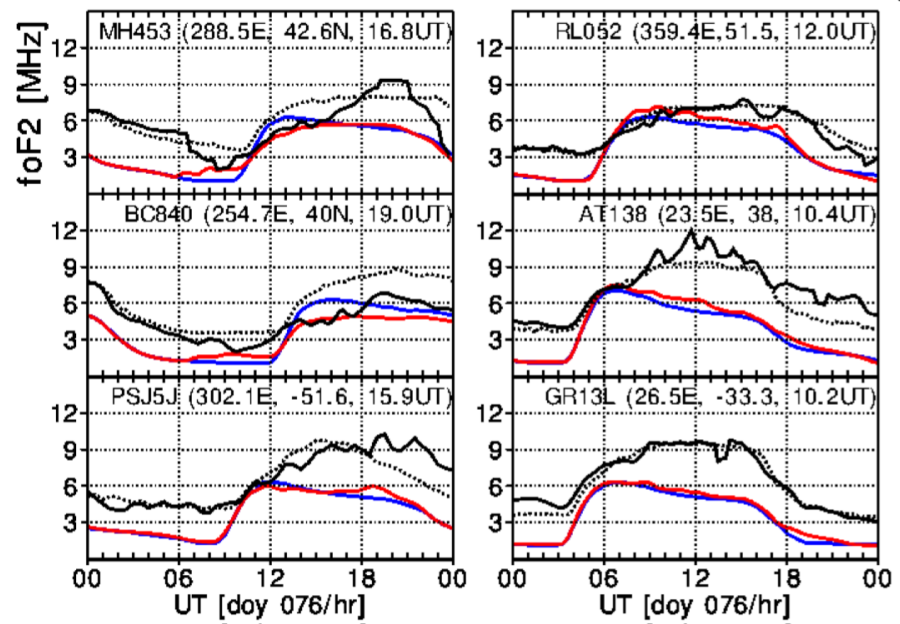

\section{TIEGCM}
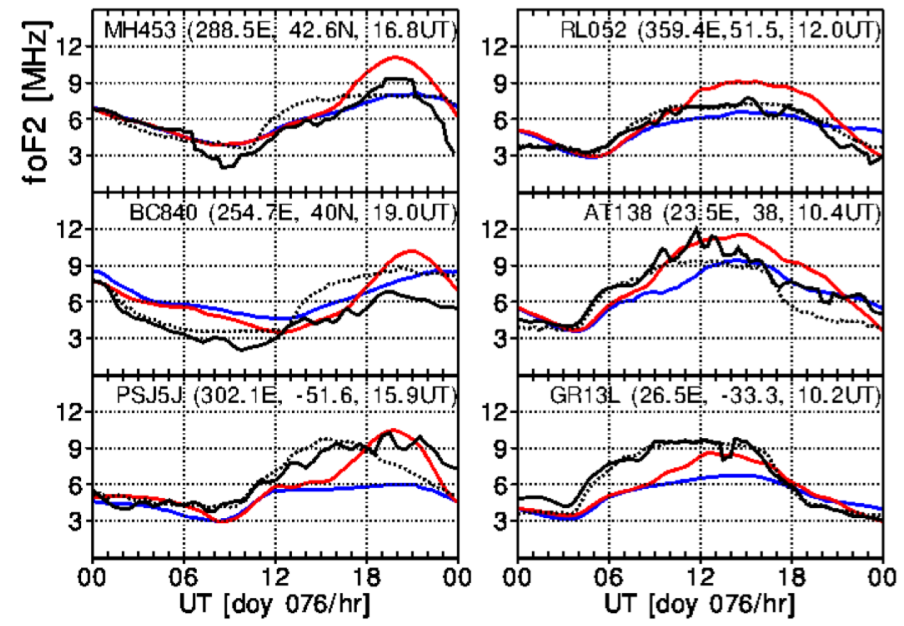

(d) 11_CTIPE
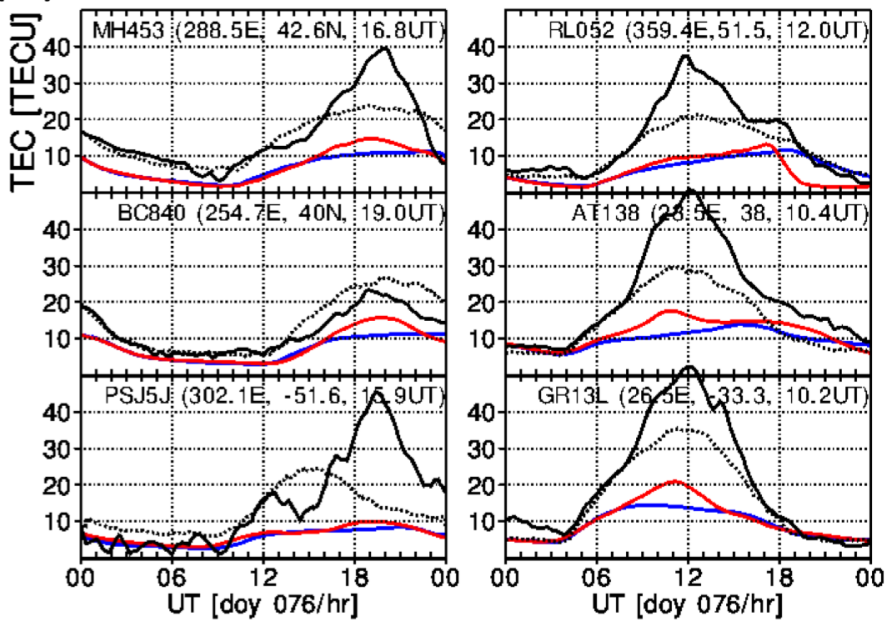

(e)

6_GITM
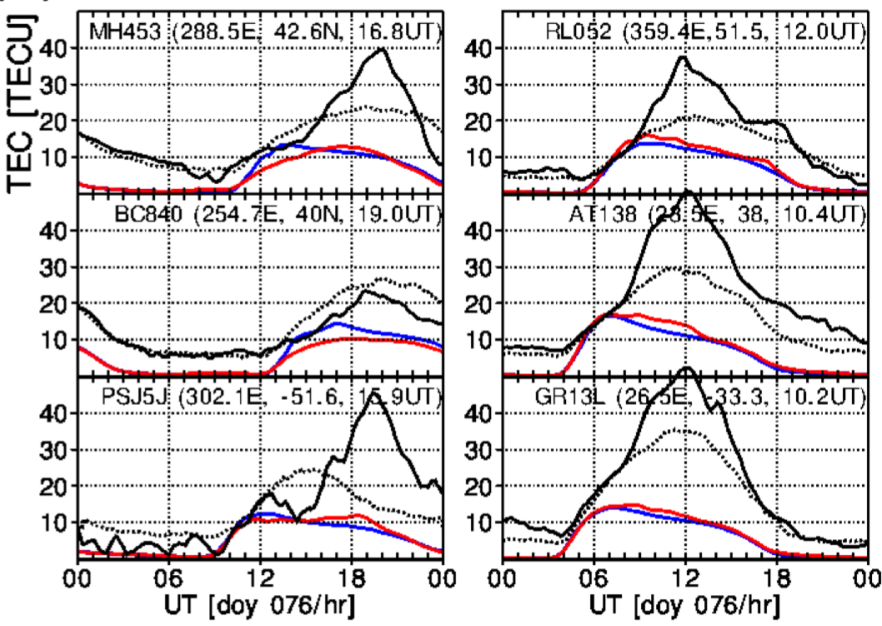

(f)

11_TIEGCM

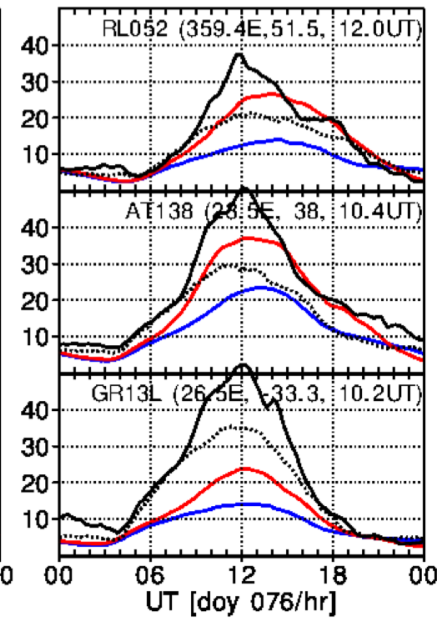


1_UAM-P
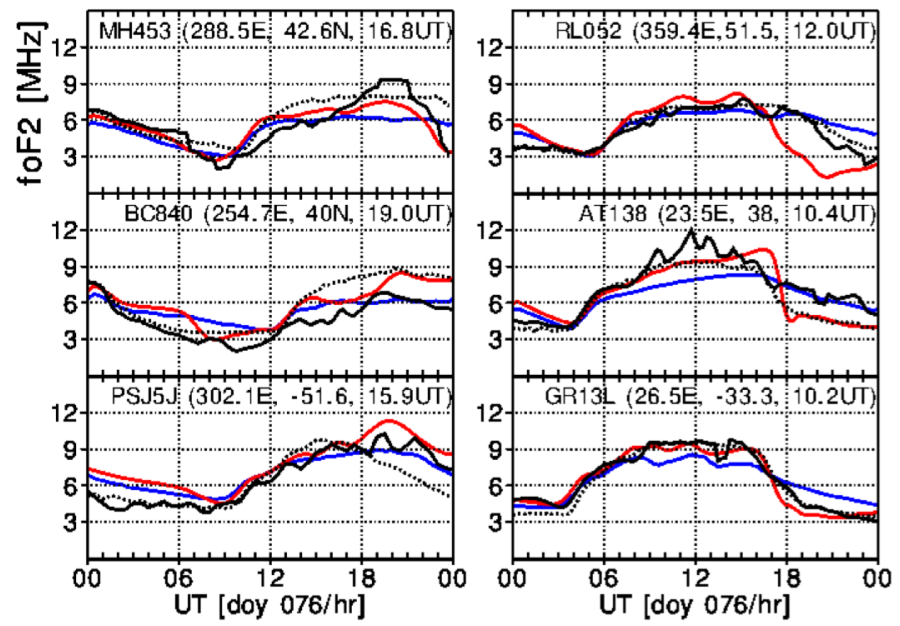

1_USU-GAIM
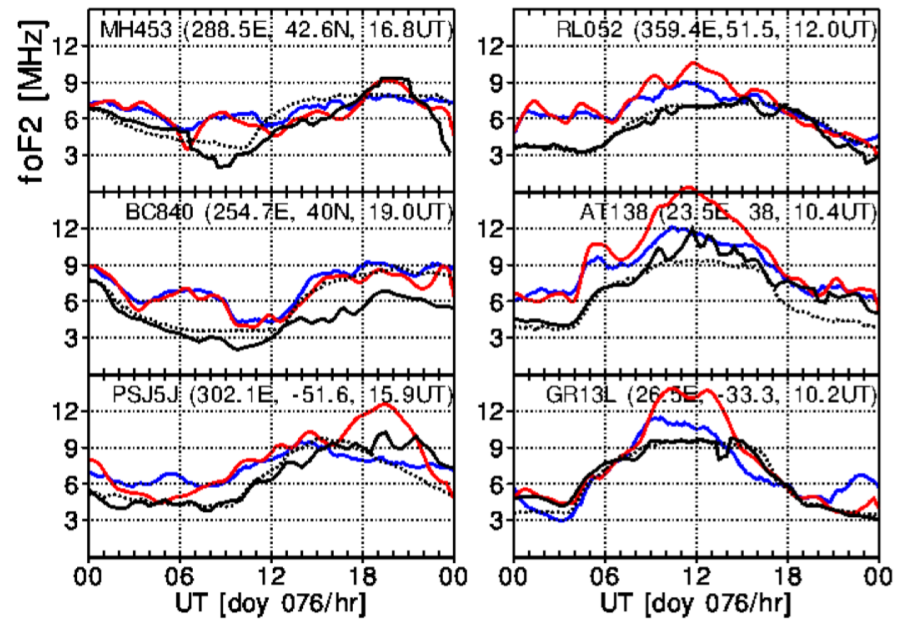

(g)

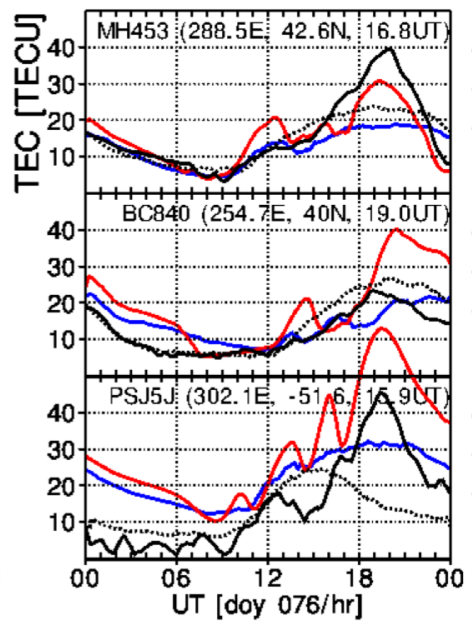

1 UAM-P

(h)

1_USU-GAIM

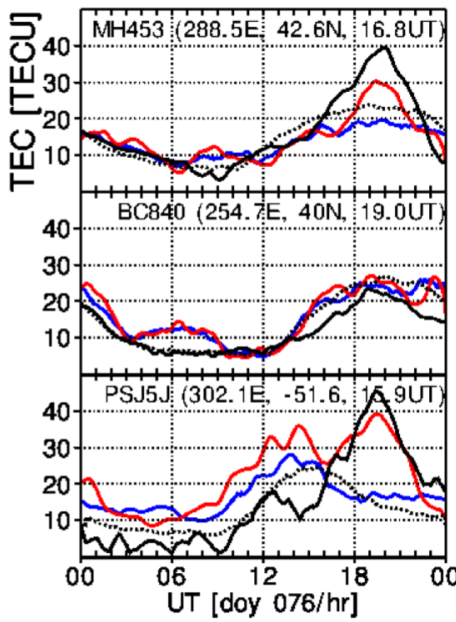

Figure S1. Comparison foF2 and TEC data with modeled values: (a) 4_IRI, (b) 1_IFM, (c) 1_SAMI3, (d) 11_CTIPE, (e) 6_GITM, (f) 11_TIEGCM, (g) 1_UAM-P, and (h) 1_USUGAIM. In each plot, foF 2 in the first two and TEC in the other two columns. Black solid and dotted lines denote observed storm time values and quiet-time reference (30-day median) respectively. Red and blue curves denote modeled storm time values and 30-day median. 


\section{Observed vs Modeled foF2}

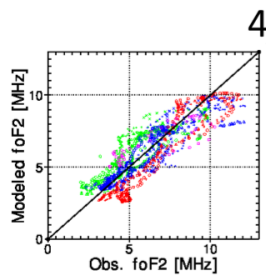

4 IRI
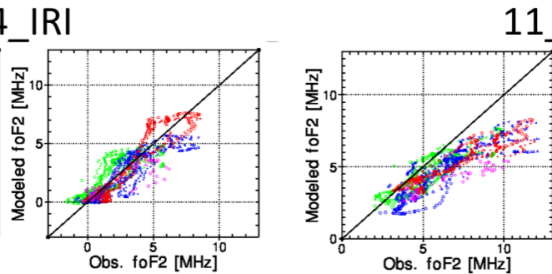

11_CTIPE

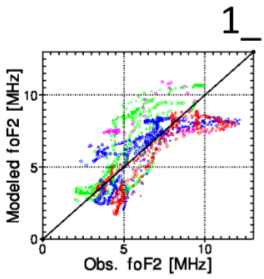

1_IFM

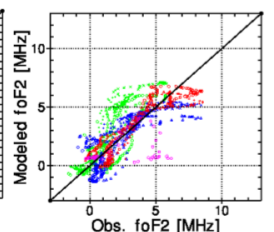

1_SAMI3
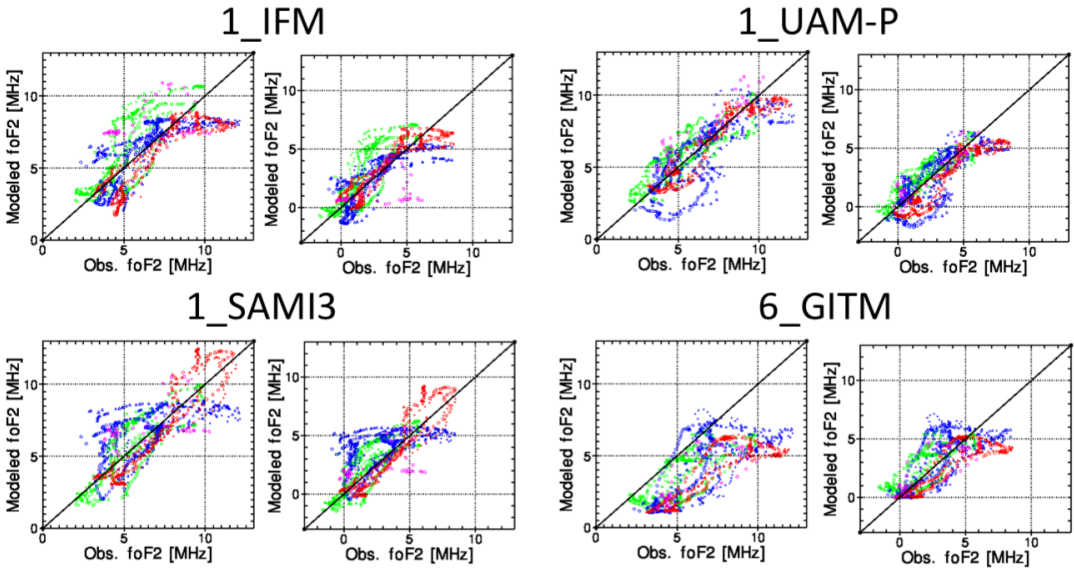

1.USU-GAIM

6_GITM
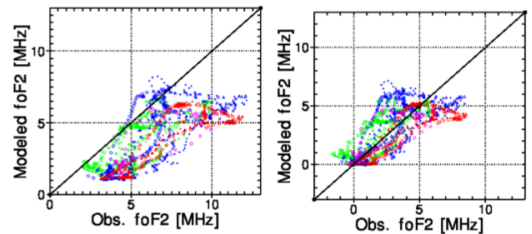

11_TIE-GCM

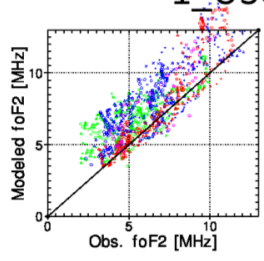

foF2

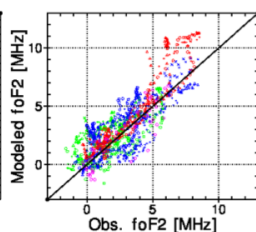

shifted foF2

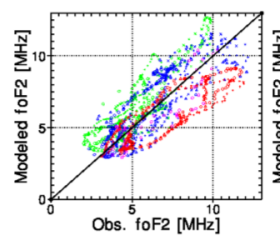

foF2

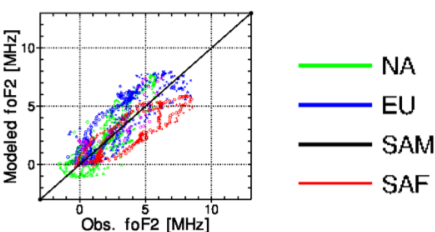

shifted foF2

Figure S2. Scatter plots of the observed (x-axis) and modeled foF2 (y-axis). For each simulation, foF2 values before and after shifting are in left and right panels, respectively. Green, blue, black and red associate with North America, Europe, South America, and South Africa, respectively. 


\section{RMSE Scatter Plot}
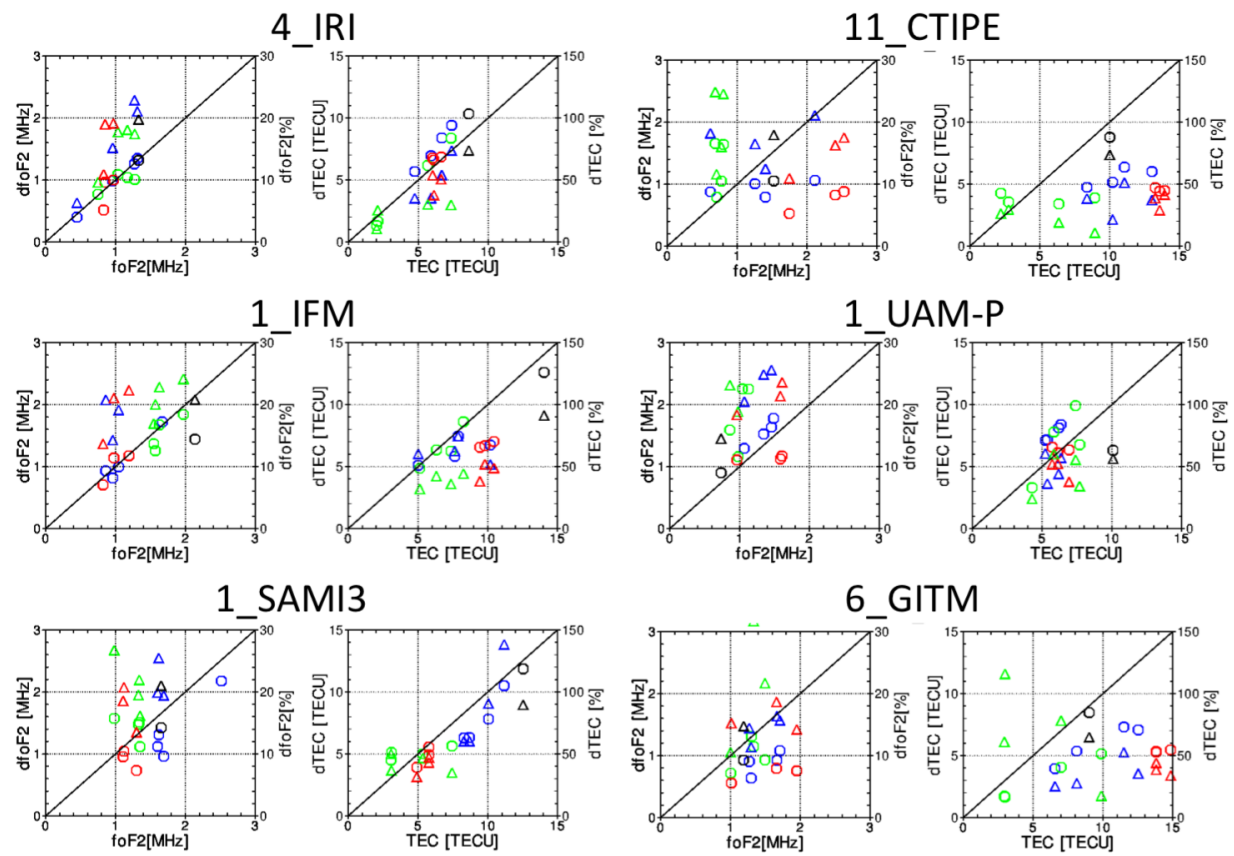

1_USU-GAIM
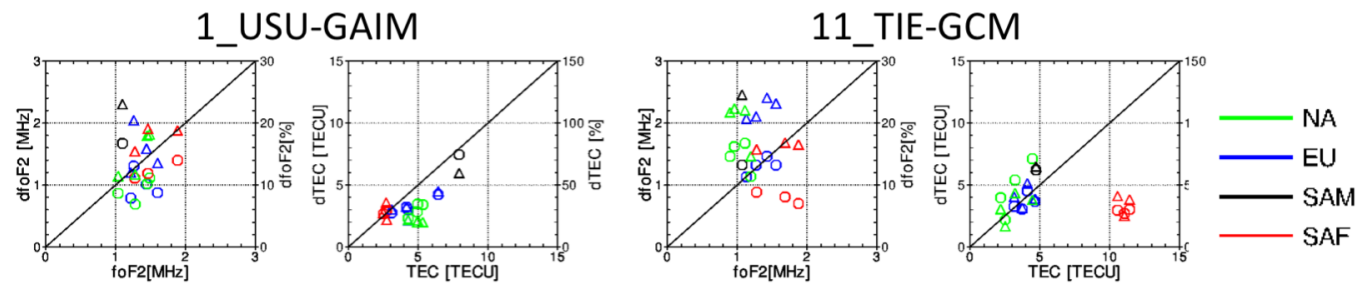

foF2 vs dfoF2 TEC vs dTEC

circles: dfoF2 or dTEC triangles: percentage changes

Figure S3. Scatter Plots of RMSE of the eight simulation. For each simulation, in left (right) panel, foF2 (TEC) on the x-axis and dfoF2 and dfoF2[\%] (dTEC and dTEC $[\%])$ on the y-axis. Green, blue, black and red associate with North America, Europe, South America, and South Africa, respectively. Circle and triangle indicate changes and percentage changes. 


\section{Yield and Timing Error Scatter Plot}

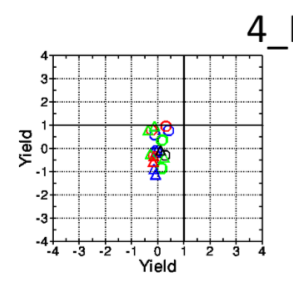

4_IRI
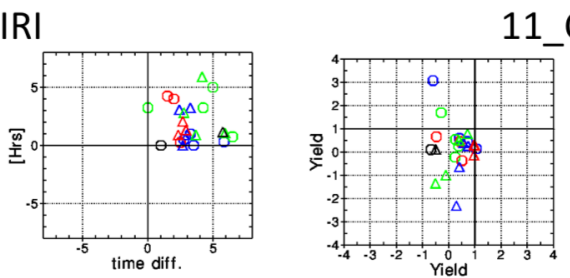

11_CTIPE

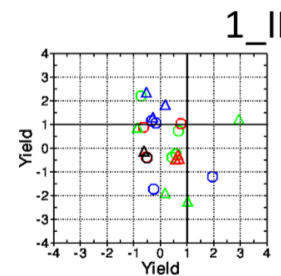

1_IFM
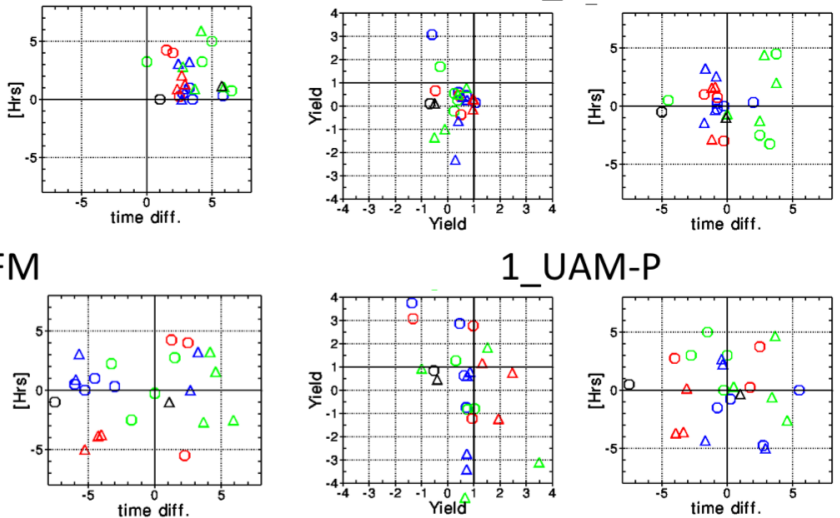

1_SAMI3
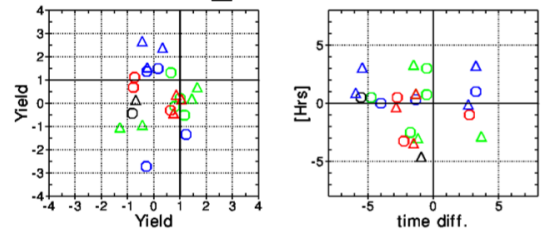

1 USU-GAIM
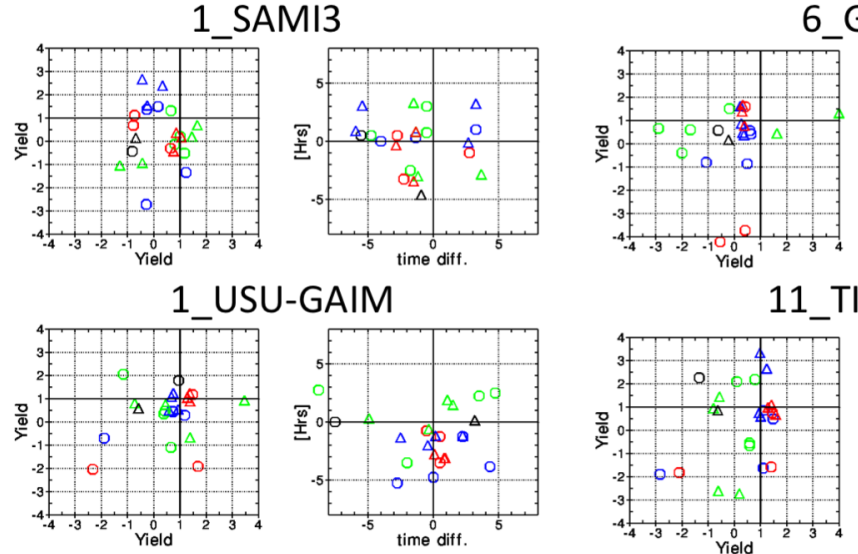

6_GITM

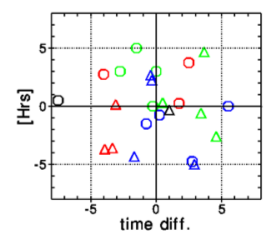

\section{1_TIE-GCM}
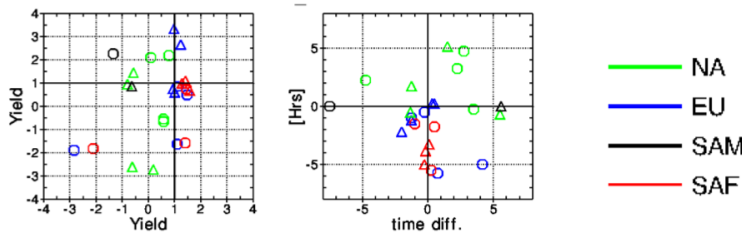

Yield

Timing Error

circles: dfoF2[\%]

triangles: dTEC[\%]

Figure S4. Scatter Plots of Yield and Timing Error (TE) of percentage changes of foF2 (circles) and TEC (triangles). For each simulation, Yield and TE are in left and right panels, respectively. Yield and TE for the time interval of 06-15UT are on the $\mathrm{x}$-axis and those of 15-22UT on the y-axis. Green, blue, black and red associate with North America, Europe, South America, and South Africa, respectively. 